\title{
Differentiation between palatal rugae patterns of twins by means of the Briñon method and an improved technique
}

\section{Lara Maria HERRERA ${ }^{(a)}$ Raíssa Ananda Paim STRAPASSON(a) Luiz Eugênio Nigro MAZZILLI(a) Rodolfo Francisco Haltenhoff MELANI(a)}

(a) University of São Paulo - USP, School of Dentistry, Departament of Social Odontology, São Paulo, SP, Brazil.
Declaration of Interests: The authors certify that they have no commercial or associative interest that represents a conflict of interest in connection with the manuscript.

\section{Corresponding Author:}

Lara Maria Herrera

laraherrera@usp.br

DOI: 10.1590/1807-3107BOR-2017.vol31.0009

Submitted: Aug 24, 2015

Accepted for publication: Nov 01, 2016

Last revision: Nov 30, 2016

\begin{abstract}
Palatal rugae patterns are anatomic structures considered unique to each person. Monozygotic twins present similarities, however, Rugoscopy in particular, may contribute to their individualization for forensic purposes. The aims of this study were: to study the palatal rugae classifications of Briñón; to propose improvements to facilitate use of this method, if pertinent; and to characterize palatal rugae in a sample of Brazilian monozygotic twins and singletons. Precise reproducibility of the two methods of Briñón, from 1982 and 2011 , was prevented by poor intra-examiner agreement $(70 \%$ and $13 \%$ respectively). Our proposed improvements to these methods, although preliminary, were associated with better results. The most common palatal rugae patterns were types A, M, and Q. Palatal rugae were confirmed to be unique to each individual, even in monozygotic twins. Furthermore, twins did not exhibit any special patterns that might facilitate their differentiation from singletons.
\end{abstract}

Keywords: Forensic Sciences; Forensic Dentistry; Forensic Anthropology; Palate; Twins.

\section{Introduction}

Palatal rugae are structures located on the palate and are formed in the third month of intrauterine life. ${ }^{1,2,3}$ In addition to aiding mastication, sense of taste, and facilitating proper placement of the tongue in the oral cavity, they may be used to establish human identity. ${ }^{1,4,5}$

The palatal ridges - their shape, position, number, and orientation - are unique to each individual. ${ }^{6,7}$ However, classification of palatal rugae still represents a challenge because of the wide range of different methodologies available and, consequently, lack of standardization, as well as the subjective nature of interpreting these characteristics. ${ }^{8}$

With the purpose of improving recording and interpretation of palatal rugae, Briñón ${ }^{9,10}$ proposed a classification in 1982 (later updated in 2011) similar to the fingerprint system. However, no studies testing or using either of these classifications have been published in the literature; thus, their practical application remains unclear.

Several studies have sought to characterize ridge patterns in different populations, ${ }^{1,5,6,7,11,12}$ which could facilitate the identification of groups in the event of a mass casualty incident. ${ }^{12}$ Research has also found that twins do 
not exhibit the same palatal rugae pattern.,6,13,14 This could be used to contribute to the their differentiation, as the similar physical characteristics of twins can confuse identification.

Within this context, the present study aimed to: study the palatal rugae classifications of Briñón;,110 propose improvements to these methods, seeking to facilitate their use, if pertinent; and characterize palatal rugae patterns in a sample of Brazilian monozygotic twins and singletons.

\section{Methodology}

\section{Study design and sample selection}

This was a cross-sectional study. The sample, selected by a non-probabilistic convenience strategy, was composed of 10 pairs of adult monozygotic twins ( 4 men and 16 women, age 20 to 34 years) and 20 adult singletons (11 men and 9 women, age 22 to 35 years), all residing in the state of São Paulo, Brazil. The first part of this research project involved only twins, while the second and the third parts involved both twins and singletons.

None of the participants had distinguishing oral clefts, lesions, inflammation, scar tissue, or grooves in the palate. Individuals that were allergic to dental impression materials were not included.

Informed consent was obtained from each participant, and all procedures were performed in accordance with the research protocol approved by the University of São Paulo School of Dentistry (FOUSP) Research Ethics Committee, under administrative procedure no. 1.014.974.

\section{Rugoscopy record}

An impression of the maxillary dental arch of each individual was taken with irreversible hydrocolloid material (alginate), and plaster casts were obtained. Palatal ridges were delineated using a graphite pencil to facilitate their visualization and later analysis. Photographic records of the casts were obtained by using two cameras (Nikon D80 and Nikon D3200) positioned parallel to the surface of the palate.

The casts were classified by groups (twins and singletons) and numbered (from 1 to 20) by computer-based randomization (<www.random.org $>$ ). The numbers were entered into a form containing the name, sex, and age of the corresponding participant.

\section{Analysis and classification of the palatal rugae}

The rugoscopic structures of the twin subjects were classified in accordance with the methods of Briñón, 9,10 as described in Figure 1. For each method, all casts were classified six times, on different days, by a single observer (standard examiner). The sixth analysis was considered as the reference patterns (RP), and intraexaminer agreement coefficients were calculated.

Then, improvements to the methods were proposed and tested for the same sample of twins, by the same examiner; intra-examiner agreement was calculated again. In addition, for a new sample of non-twin subjects, four casts were randomly selected (<www.random.org $>$ ). Two examiners performed four analyses of each cast, on different days, for a total of 16 readouts each. Percent intra- and inter-examiner agreement was calculated.

For all casts (twin and singleton subjects), rugoscopic structures were classified by using the improved method. The standard examiner performed all assessments. Data were then separated by pairs for recording and comparison. For singleton subjects, pairing was performed randomly.

\section{Results}

The classification of Briñon ${ }^{9,10}$ for analysis of the palatal rugae was evaluated. Out of 100 readouts assigned classification " $A$ ", the reproducibility of the method, assessed by means of intra-examiner agreement, was $70 \%$. When comparing readouts with the $\mathrm{RP}, 46 \%$ were in disagreement. For classification " $\mathrm{B}$ ", the reproducibility was $13 \%$. When data were compared with the RP, 93\% were in disagreement.

Our proposed improvements to the updated method of Briñón ${ }^{10}$ are listed below:

a. The accessory rugae were still assigned lower case letters and separated from the fundamental rugae by a plus sign (" + "). However, as with fundamental rugae of the same order, accessory rugae on the same "line" were also separated by periods. When there was a gap between accessory ridges in the same "line" or when these ridges were on different lines, they were assigned a plus sign; 
b. Under this new proposal, different types of rugae are no longer combined according to angulation; e.g., those previously classified as "BE" (type B rugae inclined to the left) were now classified only as "B"; c. Some rugae were incorporated into the same category by degree of similarity in shape, extension, and angulation, and, consequently, by the degree of subjectivity in differentiating them as observed in the previous study;

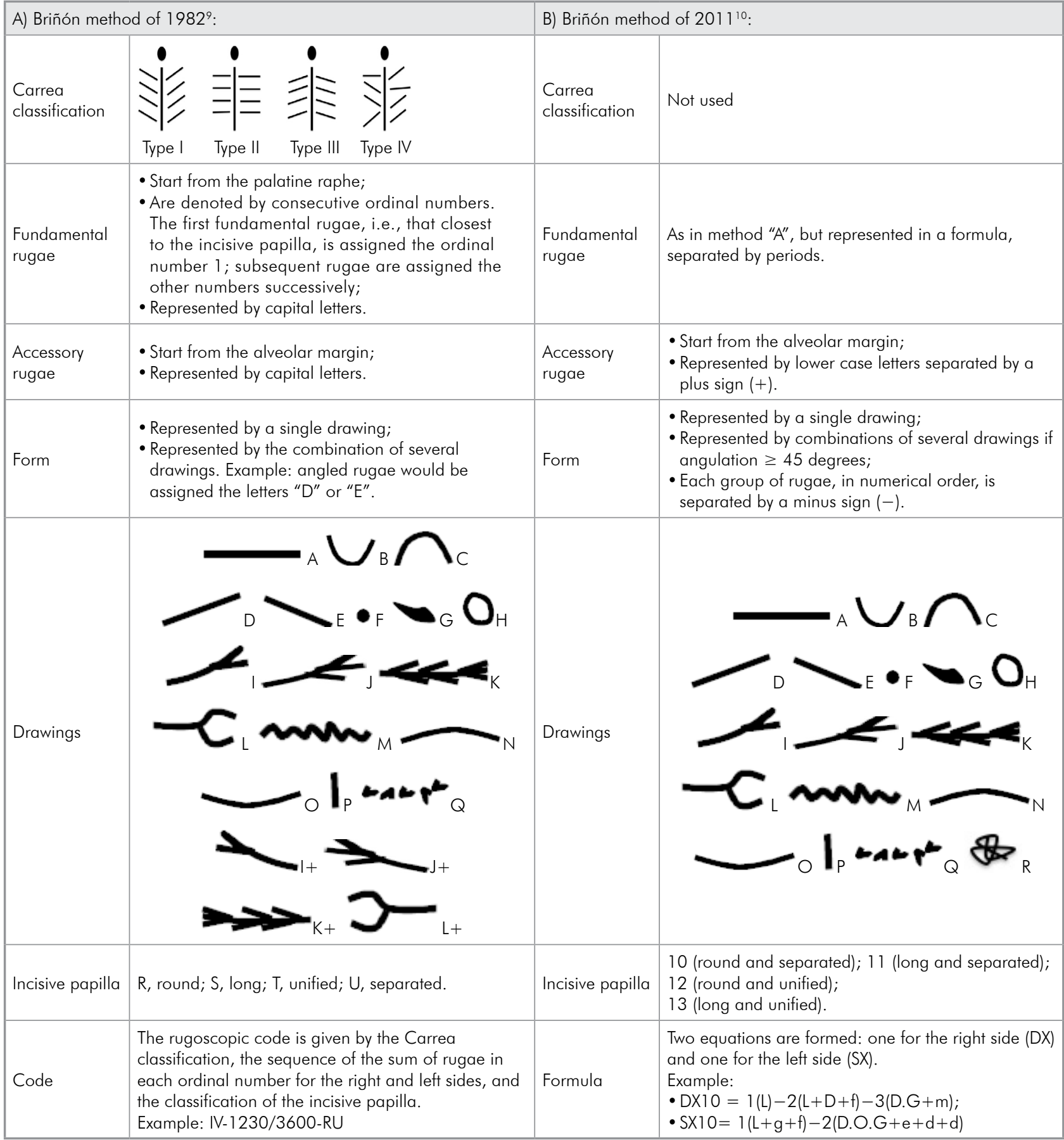

Figure 1. Briñón's classification of palatal rugae. 
d. The combinations of curved rugae used by Briñón ${ }^{10}$ were changed;

e. Some metric parameters were incorporated into the morphologic parameters in order to make interpretation of certain palatal rugae objective. These parameters are illustrated in Figure 2.

Each group of rugae, in numerical order, continued to be separated in the formula by the minus sign ("'-").
Classification of the incisive papillae also remained the same.

A total of 100 new readouts were performed using the improvements proposed. The reproducibility of the improved method, assessed by intraexaminer agreement, was $88 \%$. When comparing readouts with the $\mathrm{RP}$, of the 100 classifications obtained, 30\% were in disagreement. Table 1

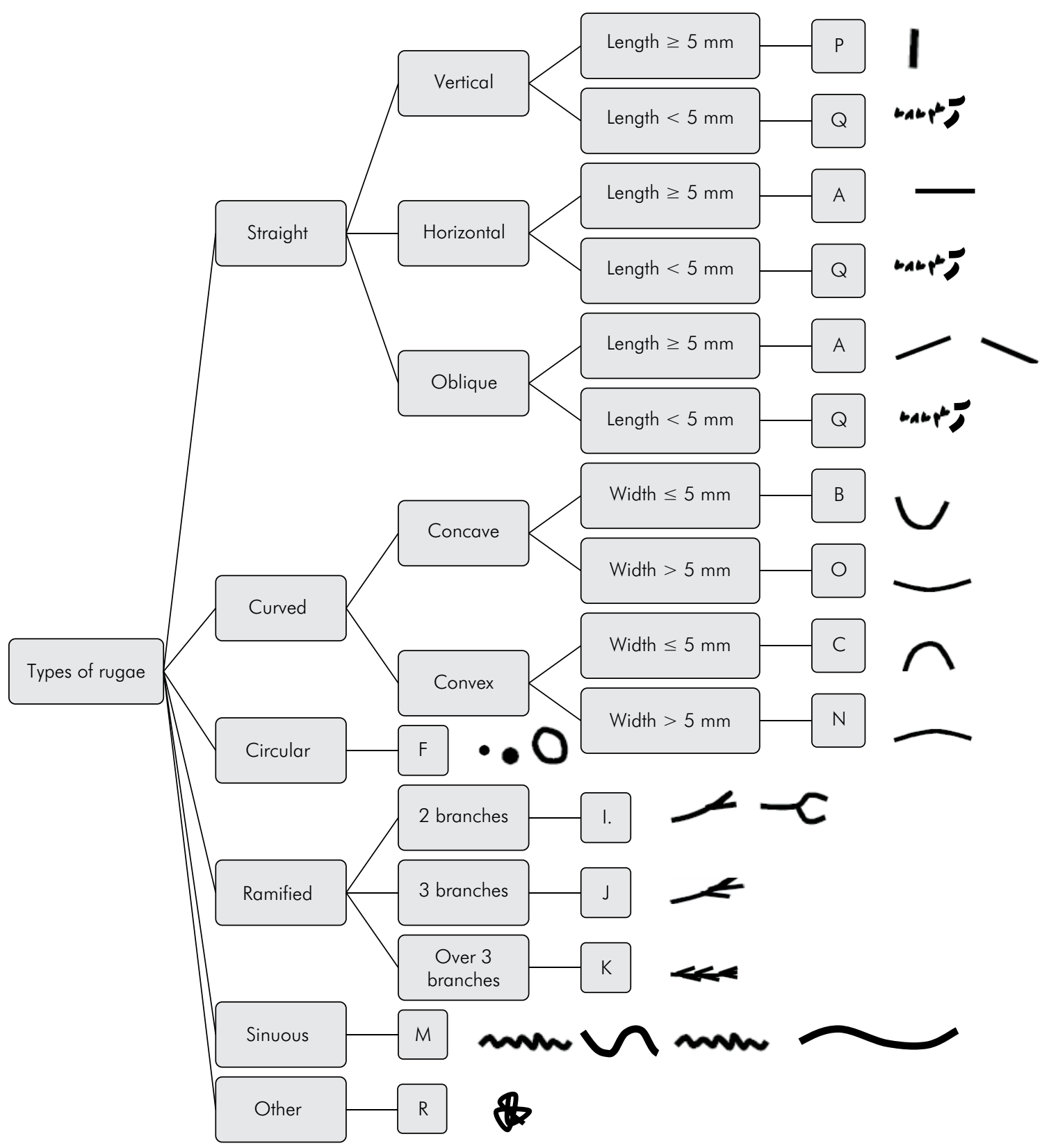

Figure 2. Proposed improvements to the 2011 Briñón classification. 
shows a comparison of results obtained with the improved method versus those obtained with the method originally proposed by Briñón in 2011 (method "B").

When the improved method was applied to a new sample of singleton subjects, its reproducibility for both examiners was $93.75 \%$, whereas the inter-examiner agreement was $25 \%$.

We observed that no individual presented the same classification, even between pairs of twins. Furthermore, the patterns found on the left side were not equal to those observed the right side.

Table 2 shows the total number of rugae and repeated occurrences when the pairs were compared. There was no statistically significant difference in frequency of repetitions of palatal rugae between groups (twins and singletons).

Table 3 presents the number of rugae, stratified by type, in twin and singleton subjects. No association was observed between types of rugae and groups ( $p>0.1)$. In this sample, the most prevalent types of rugae were $\mathrm{A}, \mathrm{M}$, and $\mathrm{Q}$.

\section{Discussion}

Rugoscopy represents a scientifically established auxiliary method of human identification. However, many classification systems are available ${ }^{15}$, and some are still very difficult to use.

In this study, palatal rugae were classified according to the schemes proposed by Briñon. ${ }^{9,10}$ To the best of our knowledge, this was the first report to scientifically evaluate these methods.

We considered the intra-examiner agreement obtained with method " $A$ " as insufficient (70\%). For method " $B$ ", intra-examiner agreement was even worse at $13 \%$, indicating that the same trained observer was unable to adequately adjust his agreement.

One of the key essential properties of any method of human identification is classifiability, i.e., it must be easy to record, amenable to filing, and allow data to be retrieved later. ${ }^{16}$ The intra-examiner agreement results obtained for our updated methodology of palatal rugae classification were not consistent with the proposal of Briñon, who restructured the original 1982 method $^{9}$ to clarify and speed up classification of palatal rugae for purposes of human identification. ${ }^{10}$

An explanation for the better reproducibility of method " $\mathrm{A}$ " is the fact that the classification generates a single final numerical code. Therefore, the types of rugae are not shown in the classification, only the number of rugae on the right and left maxillary hemiarches. For method "B", on the other hand, the description of all types of rugae in the formulas for both sides produced a greater number of variables, improving examiner error. Furthermore, classification of rugae on the basis of a chart of figures was considered highly subjective.

The subjectivity of intra- and inter-examiner observations and of the methods of classification of palatal rugae was reported as a problem by Caldas et al. ${ }^{15}$ Gondivikar et al. ${ }^{11}$ also reported subjectivity in ascertaining the shape of palatal rugae, although this parameter is extremely simple to record. The subjectivity found in this study may be due to the variations in the shape and dimension of the same type of palatal rugae in the same individual and between individuals.

The important role played by palatal ridge anatomy in human identification should not be ignored. However, as two individuals may present with the same number of rugae in the palate, method " $B$ " (which includes different shapes) would have potential for use as a classification scheme if some changes were made to reduce subjectivity and increase reproducibility.

Palatal rugae are classifiable structures. However, the schemes proposed by Briñón are complex and, as shown in this study, preclude precise reproducibility.

Table 1. Reproducibility of rugoscopic methods and classification of intra-examiner agreement.

\begin{tabular}{lccc}
\hline Method & Readouts (n) & Intra-examiner agreement (\%) & Classifications in disagreement with reference pattern (\%) \\
\hline Briñón (2011) & 100 & 13 & 93 \\
Improved Briñón (proposed) & 100 & 88 & 30 \\
\hline
\end{tabular}


Table 2. Occurrence of coincident (repeated) palatal rugae in twins and singletons.

\begin{tabular}{lcc}
\hline Variable & Twins & Singletons \\
\hline Coincident & 194 & 197 \\
Non-coincident & 47 & 51 \\
Total & 241 & 248 \\
\hline
\end{tabular}

Chi-square $=0.03 ; \mathrm{Df}=1 ; \mathrm{p}>0.1$ (not significant).

We proposed a series of changes seeking to simplify the updated Briñón classification ${ }^{10}$ and obtain better intra-examiner agreement.

The results were deemed more satisfactory in terms of reproducibility between the two examiners, as the rate of code repetition was higher than $90 \%$.

Uniformity between examiners could not be ensured, as demonstrated by the inter-examiner agreement of $25 \%$. However, when comparing classifications, the differences were perceived to be small (up to three rugae per subject). This was due to the fact that, our proposed changes notwithstanding, the number of variables was still very high. Given the subjectivity of classifying the shape of palatal rugae, this may create a sense of insecurity in the examiner.

Although there was a substantial difference between examiners, we cannot state that rugoscopy is not an effective method for identification. This is because, in forensic practice, identification is performed by comparison between antemortem and postmortem records of the palate, not by comparison of palatal rugae classifications.

Several authors ${ }^{1,5,6,7,17,18,19}$ have affirmed that the palatal rugae are unique in each individual, and the present study supports this statement. No subject presented the same set of palatal rugae, not even monozygotic twins.

As far as the uniqueness of palatal rugae in twins is concerned, these same findings were described in few studies, such as that of Ritter (apud English et al. ${ }^{20}$ ), who concluded that the pattern of palatal rugae between twins was very similar, but not identical; and Lysell, ${ }^{4}$ who verified differences and similarities in rugoscopic patterns in monozygotic and fraternal twins. Kamala et al., ${ }^{14}$ after analyzing a pair of twins and their parents, concluded that twins do not present the same palatal rugae patterns. Indira et al. ${ }^{6}$ evaluated palatal rugae patterns in five pairs of dizygotic twins and found these patterns to be different and unique in all subjects of the sample, despite some similarities at specific locations in two pairs of twins.

In the present study, on pairwise comparison, the degree of repeated occurrences of palatal rugae was similar in twins and singletons; that is, monozygotic twin pairs did not exhibit any set of repetitions that could differentiate them from the general population.

Table 3. Distribution of types of palatal rugae in twins and singletons.

\begin{tabular}{|c|c|c|c|c|c|}
\hline \multirow{2}{*}{ Type } & \multicolumn{2}{|c|}{ Number of rugae in twins $(n=241)$} & \multicolumn{2}{|c|}{ Number of rugae in singletons $(n=248)$} & \multirow{2}{*}{ Chi-square } \\
\hline & Present & Absent & Present & Absent & \\
\hline A & 62 & 179 & 53 & 195 & $1.05^{*}$ \\
\hline B & 1 & 240 & 3 & 245 & - \\
\hline C & 5 & 236 & 3 & 245 & - \\
\hline $\mathrm{F}$ & 23 & 218 & 34 & 214 & $1.67^{*}$ \\
\hline I & 25 & 216 & 19 & 229 & $0.79^{*}$ \\
\hline J & 3 & 238 & 1 & 247 & - \\
\hline K & 0 & 241 & 0 & 248 & - \\
\hline M & 43 & 198 & 57 & 191 & $1.68^{\circ}$ \\
\hline$N$ & 18 & 223 & 18 & 230 & - \\
\hline 0 & 16 & 225 & 8 & 240 & - \\
\hline$P$ & 0 & 241 & 1 & 247 & - \\
\hline$Q$ & 45 & 196 & 51 & 197 & $0.17^{*}$ \\
\hline $\mathrm{R}$ & 0 & 241 & 0 & 248 & - \\
\hline
\end{tabular}

${ }^{*} p>0.1$ (not significant). 
Moreover, the equivalence in types of rugae between the twin and singleton groups made it clear that both groups belonged to the same population.

The palatal rugae types $\mathrm{A}, \mathrm{M}$, and $\mathrm{Q}$ were the most prevalent in both groups. These results are similar to those reported in previous studies. Gondivkar et al. ${ }^{11}$, analyzing a western Indian population, found that the most common type of palatal rugae was the "sinuous" type, corresponding to type " $\mathrm{M}$ " in the present study. In a Portuguese sample studied by Santos and Caldas ${ }^{1}$, the most prevalent types of rugae were the "straight" and "wavy", corresponding to types " $\mathrm{A}$ " and " $\mathrm{M}$ " in the classification used herein. Ibeachu et al., ${ }^{12}$ in a study of Nigerians, found that the most frequent types were "wavy" rugae (corresponding to type " $\mathrm{M}$ " in our classification) in the Igbo tribe and "curved" and "straight" rugae (corresponding to types " $\mathrm{B} / \mathrm{C} / \mathrm{N} / \mathrm{O}$ " and " $\mathrm{A}$ " in our classification, respectively) in the Ikwerre people.

Some authors have suggested that the predominance of certain palatal rugae types in different population groups can be used for identification purposes.,11 Our study does not bear out this proposal, probably due to the heterogeneity in classifications used in different studies, which make it impossible to perform a more faithful comparison.

\section{Conclusion}

A degree of subjectivity was observed when the Briñón methods ${ }^{9,10}$ were used for classification of palatal rugae. The methods were not reproduced precisely. However, the more recent version (2011) demonstrated potential for use as a classification reference, as it takes into account type rather than number of rugae.

The satisfactory percentage of repetitions of palatal rugae classifications (intra-examiner agreement) suggests that the improvements to the 2011 Briñon classification ${ }^{10}$ proposed in this study, although preliminary, do contribute to the achievement of better results. Further investigation is needed to ascertain the effectiveness of the improved method as compared with other palatal rugae classifications proposed in the literature.

Palatal rugae are unique to each individual, even in monozygotic twins, who, in this study, did not exhibit any special rugae patterns that could differentiate them from other groups. Nevertheless, studies in larger samples are suggested.

\section{Acknowledgments}

The authors thank the volunteers for their participation in the study and the reviewers for their observations.

\section{References}

1. Santos C, Caldas IM. Palatal rugae pattern in a Portuguese population: a preliminary analysis. J Forensic Sci. 2012;57(3):786-8. doi:10.1111/j.1556-4029.2011.02016.x

2. Tornavoi DC, Silva RHAD. Rugoscopia palatina e a aplicabilidade na identificação humana. Saúde Ética Justiça. 2010;15(1):28-34. doi:10.11606/issn.2317-2770.v15i1p28-34

3. Santos KC, Fernandes CMS, Serra MC. Evaluation of a digital methodology for human identification using palatal rugoscopy. Braz J Oral Sci. 2011;10(3):199-203.

4. Lysell L. Plicae palatinae transversae and papilla incisiva in man: a morphologic and genetic study. Acta Odontol Scand. 1955;13(suppl 18):5-137.

5. Saraf A, Bedia S, Indurkar A, Degwekar S, Bhowate R. Rugae patterns as an adjunct to sex differentiation in forensic identification. J Forensic Odontostomatol. 2011;29(1):14-9.
6. Indira A, Gupta M, David MP. Usefullness of palatal rugae patterns in establishing identity: preliminary results from Bengaluru city, India. J Forensic Dent Sci. 2012;4(1):2-5. doi:10.4103/0975-1475.99149

7. Dawasaz AA, Dinkar AD. Rugoscopy: predominant pattern, uniqueness, and stability assessment in the Indian Goan population. J Forensic Sci. 2013;58(6):1621-7. doi:10.1111/1556-4029.12190

8. Kesri R, Das G, Tote J, Thakur P. Rugoscopy: science of palatal rugae: a review. Int J Dent Med Res. 2014;1(4):103-7.

9. Briñón EN. Rugas palatinas. In: Briñón EN. Odontología legal y práctica forense. Buenos Aires: Purinson; 1982. p. 291-317.

10. Briñón EN. Palatograma legal programado: modificado por su autora, Doctora Elida Norma Briñón. Rev Circ Argent Odontol. 2011;68(212):11-5. 
11. Gondivkar SM, Patel S, Gadbail AR, Gaikwad RN, Chole R, Parikh RV. Morphological study of the palatal rugae in western Indian population. J Forensic Leg Med. 2011;18(7):310-2. doi:10.1016/j.jflm.2011.06.007

12. Ibeachu PC, Didia BC, Arigbede AO. A comparative study of palatal rugae patterns among Igbo and Ikwerre Ethnic Groups of Nigeria: aUniversity of Port Harcourt Study. Anat Res Int. 2014;2014:ID123925. doi:10.1155/2014/123925

13. Fahmi FM, Al-Shamrani SM, Talic YF. Rugae pattern in a Saudi population sample of males and females. Saudi Dent J. 2001;13(2):92-5.

14. Kamala R, Gupta N, Bansal A, Sinha A. Palatal Rugae Pattern as an Aid for Personal Identification: a forensic study. J Indian Acad Oral Med Radiol. 2011;23(3):173-8.

15. Caldas IM, Magalhães T, Afonso A. Establishing identity using cheiloscopy and palatoscopy. Forensic Sci Int. 2007;165(1):1-9. doi:10.1016/j.forsciint.2006.04.010
16. Turano LM, Silva M. Noções de dactiloscopia. In: Silva M. Compêndio de odontologia legal. Rio de Janeiro: Medsi; 1990. p. 161-6.

17. De Angelis D, Riboli F, Gibelli D, Cappella A, Cattaneo C. Palatal rugae as an individualising marker: reliability for forensic odontology and personal identification. Sci Justice. 2012;52(3):181-4. doi:10.1016/j.scijus.2011.09.002

18. Shetty D, Juneja A, Jain A, Khanna KS, Pruthi N, Gupta A et al. Assessment of palatal rugae pattern and their reproducibility for application in forensic analysis. J Forensic Dent Sci. 2013;5(2):106-9. doi:10.4103/0975-1475.119775

19. Barbieri AA, Scoralick RA, Naressi SC, Moraes ME, Daruge E Jr, Daruge E. The evidence of the rugoscopy effectiveness as a human identification method in patients submitted to rapid palatal expansion. J Forensic Sci. 2013;58(S1):S235-8. doi:10.1111/j.1556-4029.2012.02263.x

20. English WR, Robison SF, Summitt JB, Oesterle LJ, Brannon RB, Morlang WM. Individuality of human palatal rugae. J Forensic Sci. 1988;33(3):718-26. doi:10.1520/JFS12479J 